\title{
Controlling of Spin Wave Propagation in a Magnonic Waveguide with Alternative Antidot Width Modulation
}

\author{
Vivek $\mathrm{T}^{1}$, Zahraa Batool $^{1}$ and Sabareesan $\mathrm{P}^{1}$ \\ ${ }^{1}$ Centre for Nonlinear Science and Engineering, \\ School of Electrical and Electronics Engineering, SASTRA University, Thanjavur, Tamil Nadu. India
}

\begin{abstract}
Controlling of spin wave propagation in a 1-D Magnonic Antidot Waveguides (MAWs) is studied by introducing the periodic modulation along width of alternative antidots. In the present work, the magnonic band gaps are tuned by varying the width of alternative antidot in magnonic waveguide. The allowed band and forbidden bands are formed because of constructive and destructive interference between initial spin wave mode and higher excited quantized spin wave mode. The frequencies of the spin waves are in the order of microwave regime paves the way for building novel magnonic devices in mere future as well as quite applicable in the microwave communications.
\end{abstract}

Keywords: Magnonics,Magnonic band gap, MAWs, Spin Wave

\section{Introduction}

In modern magnetism, the evolution of magnonics intends to investigate the behaviour of spin waves in nano-structured elements. The controlling of spin waves (called magnons) which are collective excitations of individual spins in ordered magnetic material Sang-Koog Kim[1], have attracted interest in nanofabrication technologies. In semiconductor devices, the electronic band structures observed due to the periodicity in atomic structures. Similarly in photonic crystals, the electromagnetic wave propagation is controlled due to the artificially fabricated periodic structures is also issue of growing interest in condensed matter physics. The magnetic counter part of photonic crystal is the magnonic crystal. B. Lenk et.al [2] Recently, many theoretical and experimental studies have done on the magnonic crystals containing 1-D structures, such as periodic multilayers, periodic arrays of nanostrips, corrugated films, comb- like or serial loop structures and even 2-D or 3-D structures. In such assemblies, the band structures of spin wave modes are controlled by periodic structures artificially fabricated with different magnetic material parameters, shapes and exchange-bias field. Recently, Dmitriviet.al [3] proposed micromagnetic simulation for planar magnetic waveguide where studied the propagation of spin waves in MCs. Qi.Wang et.al [4 ] observed magnonic band gap by phenomena of Bragg reflection of spin waves, adjusting the distance between rectangular holes and width direction in a magnonic waveguide with periodic rectangular shaped holes. In this work, we simulated the magnonic alternative antidot waveguide that can be used for promising magnonic devices such as microwave communication. These MAWs simply composed of discrete alternative antidot with different width modulation in a nanostrip from a soft magnetic material [eg: permalloy (Py)]. The physics of occurrence of magnonics band gap with different width modulation and the allowed bands and forbidden bands of spin wave S.A. Nikitov et.al [5] are studied with respect to width modulation of alternative antidot using numerical micromagnetic simulation such as OOMMF software M. Donahue et.al [6].

\section{Sample Description}

Fig. 1 shows the schematic representation of a $2400 \mathrm{~nm}$ x $24 \mathrm{~nm}$ MCW with $3 \mathrm{~nm}$ thickness. The entire structure consists of permalloy $\left(\mathrm{Ni}_{80} \mathrm{Fe}_{20}\right)$ MAWs with alternative air holes width [W1 W2] modulation

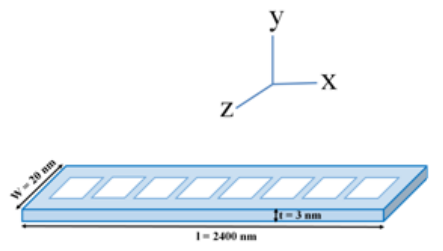

(a)

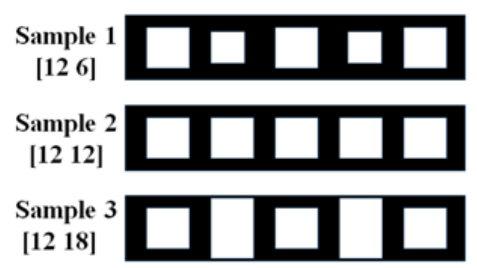

(b)

Figure 1: (a) Schematic sketch of magnonic antidot waveguide (MAW) and (b) different periodic width $\left[\mathrm{W}_{1}\right.$ $\mathrm{W}_{2}$ ] modulation of (MAWs) for constant length $12 \mathrm{~nm}$.

National Conference on Current Advancements in Physics $3^{\text {rd }} \& 4^{\text {th }}$ February 2017

Department of Physics, St. John's College, Palayamkottai-627 002, Tamilnadu, India. DOI 10.9790/4861-17002038791 
Such as [12 6] [12 12] and [12 18] as shown in Fig.1. Simulations were performed on MCWs using micro magnetic simulation software (OOMMF) to solve the Landau-Lifshitz-Gilbert (LLG) equation. To excite the different modes of spin waves by applying "sine cardinal" ( $\operatorname{sinc}$ ) pulse with bias field of $\mathrm{H}_{0}=10.1 \mathrm{kOe}$. The field was applied along the $\mathrm{x}$ direction $\left(2400 \times 24 \times 3 \mathrm{~nm}^{3}\right)$ indicated by dark colour in Fig. 1. The controlling of magnonic band gap in MAWs, due to the variation in periodic alternative antidot width modulation. The material parameters for Py nanostripe is shown in table (1) Qi.Wang et.al [4]

Table 1: Experimentally observed material parameters for the permalloy $\left(\mathrm{Ni}_{80} \mathrm{Fe}_{20}\right)$

\begin{tabular}{|c|c|}
\hline Parameters & Value \\
\hline $\begin{array}{c}\text { Gyromagnetic ratio } \\
(\gamma)\end{array}$ & $2.21 \times 10^{5} \mathrm{~mA}^{-1} \mathrm{~s}^{-1}$ \\
\hline $\begin{array}{c}\text { Gilbert damping } \\
(\alpha)\end{array}$ & 0.0001 \\
\hline $\begin{array}{c}\text { Saturation } \\
\text { Magnetization }\left(\mathrm{M}_{\mathrm{s}}\right)\end{array}$ & $800 \times 10^{3} \mathrm{Am}^{-1}$ \\
\hline
\end{tabular}

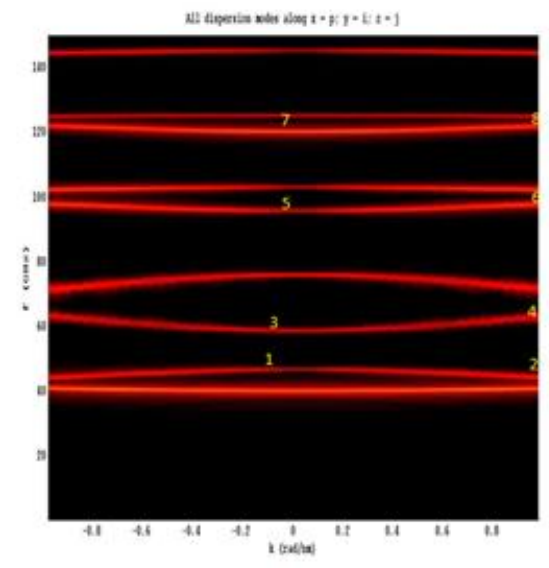

[12 6]

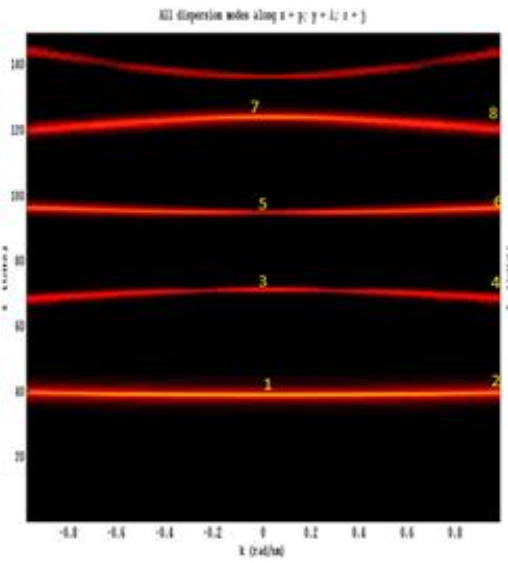

[12 12]

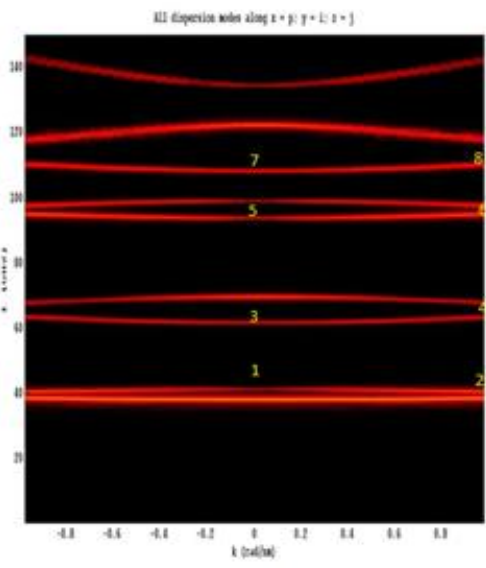

[12 18]

Figure 2: Dispersion curves for corresponding width modulation [12 6] [12 12] and [12 18] in MAWs

\section{Results And Discussion}

The dispersion curves obtained for different periodic width modulation [12 6] [12 12] and [12 18] as shown in Fig 2. Using 2-D Discrete Fourier Transform, of the results obtained from OOMMF code which solves the Landau-Lifshiftz-Gilbert (LLG) equation. The dispersion curves clearly show the allowed and forbidden bands of spin waves propagating through the MAWs. The presence of magnonic band gap in magnonic crystals due to different magnetic materials, shapes and exchange-bias field. In this work, we studied the controlling of spin waves in MAWs by changing the width in periodic alternative antidot which gives the magnonic band gap. The origin of the presence of allowed bands and forbidden bands due to width confinement (Ki-Suk Lee et.al [7]) in alternative antidot in MAWs were found by micromagnetic numerical simulation.

From the dispersion curves, there is no forbidden band expect for below the intrinsic potential barrier $(40 \mathrm{GHz})$ for different periodic width $\left[\mathrm{W}_{1} \mathrm{~W}_{2}\right]$, owing to quantization of lowest spin wave modes and its width confinement. The occurrence of $1^{\text {st }}$ mode in different width modulation [12 6] [12 18] is occurred due to interaction of initial spin wave propagation and its reflection from the antidot which generates a demagnetization field (Sang-Koog Kim et.al[8]) The second mode (backward volume mode) appeared in [12 6] [12 12] is due to the destructive interference of initial spin waves between initial spin wave mode and higher excited quantized mode. Similarly $3^{\text {rd }}$ mode (forward volume mode) in [12 6] [12 12] is due to constructive interference of spin waves. As compared to [12 6] [12 12], there is wide band gap observed in [12 12] and also reduces the modes due to destructive interference. The three forbidden band gap in [12 12] is further reduced by varying the width of the alternative antidot in MAWs. The first forbidden bands for different width modulation 
[12 6] [ $\left[\begin{array}{ll}12 & 12\end{array}\right]$ and [12 18] shown in table (2). We observed the frequency range varied for different width modulation $\left[\mathrm{W}_{1} \mathrm{~W}_{2}\right]$ revealed the controlling of spin waves in MAWs achieved by varying width modulation in alternative antidot. Further, additional modes occurred in [12 6] [ $\left.\begin{array}{lll}12 & 18\end{array}\right]$ between the $1^{\text {st }}$ and $2^{\text {nd }}$ mode due to constructive (forward volume mode) and destructive (backward volume mode) interference between the initial spin wave mode and higher quantized spin wave mode.

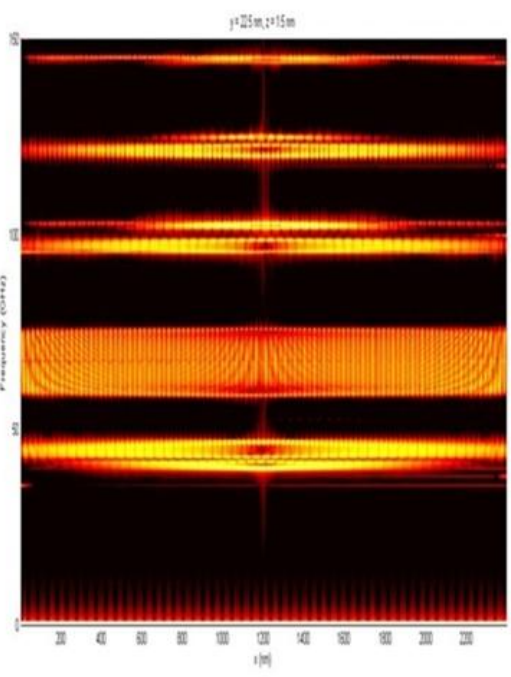

[12 6]

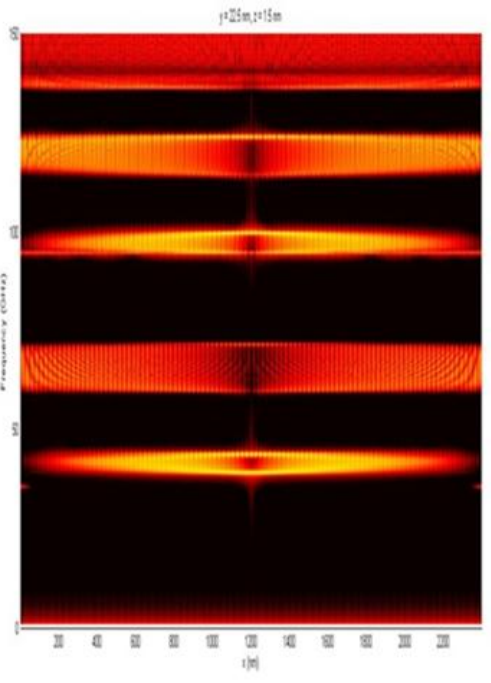

[12 12]

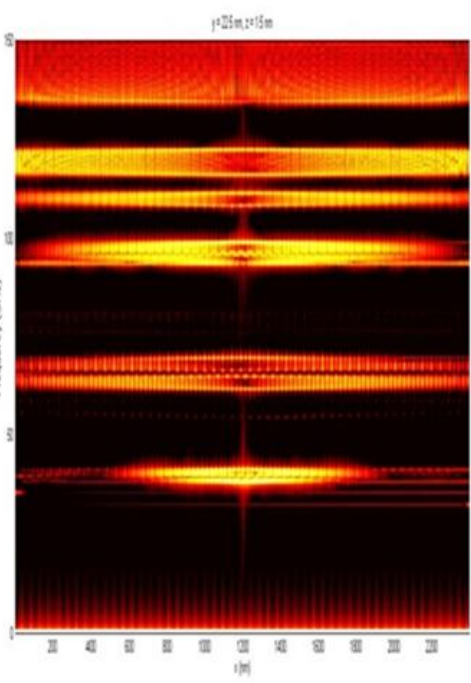

[12 18]

Figure 3: Frequency spectrum for corresponding width modulation [12 6] [12 12] and [12 18] in MAWs

Fig. (2) Shows the frequency map corresponding to the spin wave dispersion relation for periodic modulation along the width of the alternative antidots [6 12] [12 12] and [18 12]. The frequency spectrum is plotted which describes the propagation of spin waves is along the length of the MAWs. The black colour region denoted as forbidden frequency gap (band gap) and red colour region known as allowed frequencies (bandwidth) in the frequency map. From frequency map, we observed the spin wave propagation controlled by altering the width of the alternative antidot. As seen in [12 12] the band gap (black colour region) between $2^{\text {nd }}$ and $3^{\text {rd }}$ mode is wide compared to [6 12] [ 18 12]. There is destructive interference take places in [12 12] between the initial spin wave mode and higher excited quantized mode. Due to width confinement there is change in band gap in samples [6 12] [18 12]. The corresponding allowed frequencies in the range of $\mathrm{GHz}$ for periodic modulation of the alternative antidot width as given in the table (2).

\begin{tabular}{|c|c|c|c|c|}
\hline $\begin{array}{c}\text { Width of the } \\
\text { antidot (nm) }\end{array}$ & $\begin{array}{c}\text { Bandwidth 1 } \\
(\mathbf{G H z})\end{array}$ & $\begin{array}{c}\text { Bandwidth 2 } \\
(\mathbf{G H z})\end{array}$ & $\begin{array}{c}\text { Bandwidth 3 } \\
\mathbf{( G H z )}\end{array}$ & $\begin{array}{c}\text { Bandwidth 4 } \\
(\mathbf{G H z})\end{array}$ \\
\hline 6 & $38-43$ & $60-71$ & $95-100$ & $115-125$ \\
\hline 12 & $40-47$ & $59-76$ & $96-104$ & $120-126$ \\
\hline 18 & $38-42$ & $62-70$ & $94-99$ & $109-112$ \\
\hline
\end{tabular}

Table 2: The corresponding allowed frequencies in the range of $\mathrm{GHz}$ for periodic modulation of the alternative antidot width [12 6] [12 12] and [12 18] 


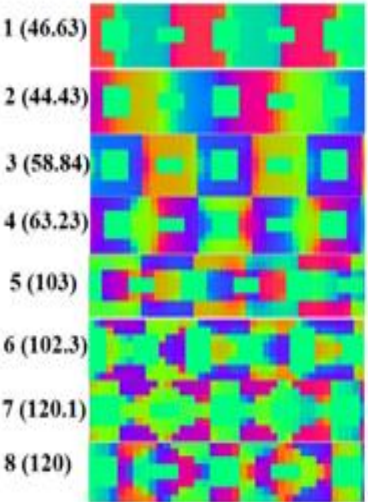

Phase

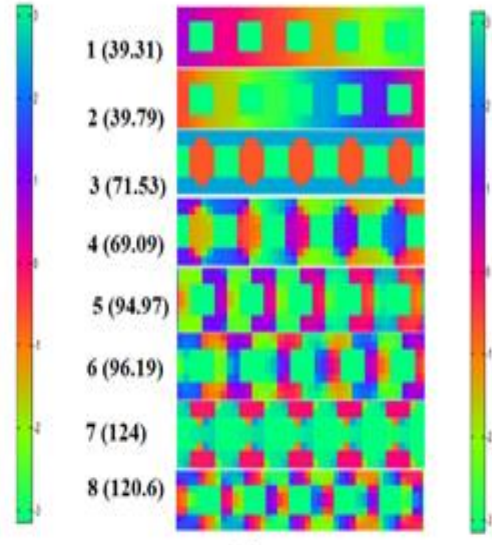

Phase

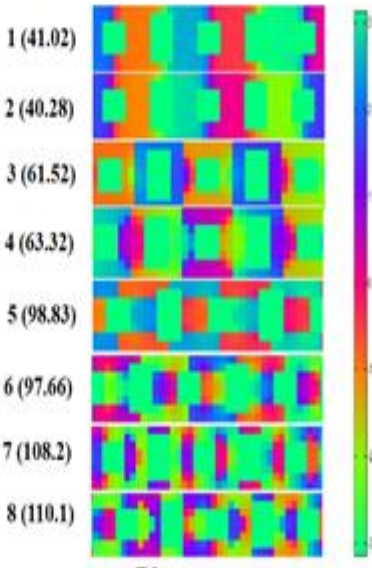

Phase

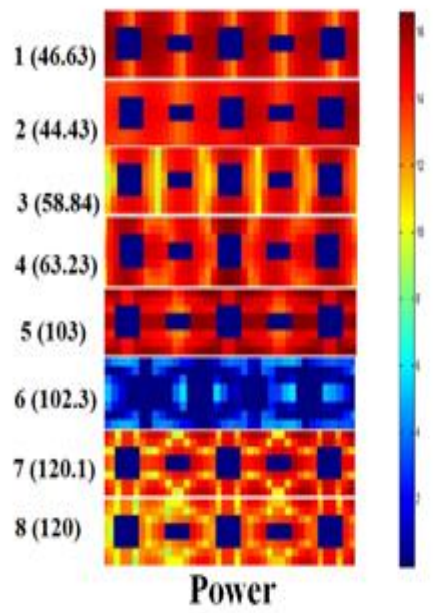

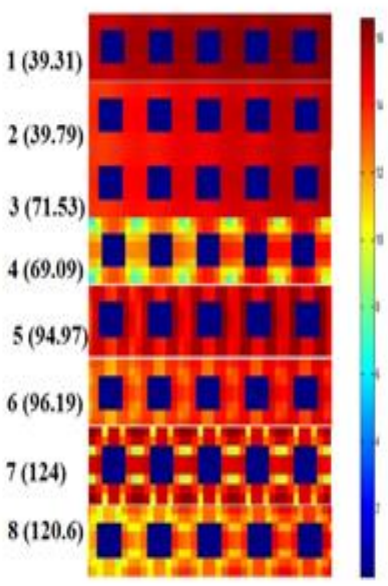

Power

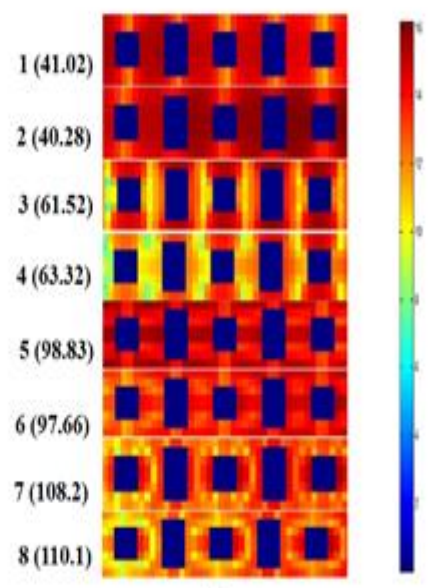

Power

Figure 4: Phase and Power Distribution Profile (PPDPs) for different width modulation [12 6] [12 12] and [12 18] in MAWs.

The above figure depicts the spatial spin wave PPDPs for the marked $(\mathrm{k}, \mathrm{f})$ values in the dispersion plot as discussed in Fig 2. The power distribution profile (PoDP) of modes (1) (2) and (3) showing insignificant power loss (i.e) the power variation is zero in [12 12] sample. PoDP of modes (1) and (2) in [12 6]and [12 18] shows the vertical nodal lines (i.e) parallel to the local direction of applied magnetic field in ' $y$ ' direction confirmed the type of spin wave propagation is backward volume (BV) as shown in Fig 2. The corresponding Phase Distribution profiles (PhDP) of modes (1) (2) and (3) in [12 6] [12 12] and [12 18] displayed in the Fig (4). PoDP of modes (3) and (4) in [12 6] and [12 18] displays the horizontal nodal lines (i.e) perpendicular of applied bias field settled the spin wave propagation is forward volume (FV) mode, but PoDP of mode (4) in [12 12] is backward volume, same physics occurred in modes (1) (2) and (3) in sample of [12 12]. The modes (7) and (8) in [12 6] and [12 18] showing the nodal lines are localized at the edge of antidot as shown in PoDP described the SW propagation type is end mode. We can also observed the band gap opened at the first Brillouin zone boundary $(\pi / \mathrm{a})$, because the spin wave propagation is in end mode due to its width confinement. In [12 12] modes (7) and (8) showed the SW propagation is BV mode as we can see in both in PoDP and dispersion plot. The corresponding PhDP of entire modes in [12 6] [12 12] and [12 18] displayed in Fig (4). Hence, we identified the types of modes for different width modulation [12 6] [12 12] and [12 18] using results of Phase and Power Distribution Profile (PPDP).

\section{Conclusions}

From the above studied, we concluded that the propagation of spin waves can be controlled by tuning the alternative antidot width modulation in magnonic antidot waveguide (MAWs) reduced the forbidden band gap and increase allowed frequencies range for our desired applications. It has alluring potentialities over 
magnonic devices in microwave applications such as logic gates, logic circuit and filters.

\section{Acknowledgements}

T.V and P.S thank SASTRA University and DST-SERB project for their financial assistantship (SB/FTP/PS$061 / 2013)$

\section{References}

[1]. Sang-Koog Kim, Micromagnetic computer simulations of spin waves in nanometre-scale patterned magnetic elements,Journal of Physics D:Applied Physics, 264004,2010, 25pp

[2]. B. Lenk, H. Ulrichsa, F. Garbs, M. Munzenberg, The building blocks of magnonics ,

[3]. G. Venkat, D. Kumar, M. Franchin, O. Dmytriiev, M. Mruczkiewicz, H. Fangohr, A. Barman, M. Krawczyk, andA. Prabhakar, Proposal for a standard micromagnetic problem:Spin wave dispersion in a magnonic waveguide, IEEE TRANSACTIONS ON MAGNETICS, VOL. 49, NO. 1, JANUARY 2013

[4]. Qi Wang, Zhiyong Zhong, Lichuan Jin, Xiaoli Tang, Feiming Bai, Huaiwu Zhang ,Geoffrey and S.D. Beach, Design of nanostrip magnonic crystal waveguides with a single magnonic band gap, Journal of Magnetism and Magnetic Materials 340, 2013, 23-26

[5]. S.A. Nikitova, Ph. Tailhadesa,and C.S. Tsai, Spin waves in periodic magnetic structures - magnonic crystals, Journal of Magnetism and Magnetic Materials 23,2001, 320-330

[6]. M. Donahue and D. Porter, “OOMMF user's guide, version 1.0,"National Institute of Standards and Technology, Gaithersburg, MD, Tech.Rep., 1999.

[7]. Ki-Suk Lee, Dong-Soo Han and Sang-Koog Kim, Physical Origin and Generic Control of Magnonic Band Gaps of Dipole-Exchange Spin Waves in Width-Modulated Nanostrip Waveguides,Physical Review Letters 102,2009 127202

[8]. Sang-Koog Kim, Ki-Suk Lee and Dong-Soo Han, A gigahertz-range spinwave filtercomposed of width-modulated nanostrip magnonic-crystal wavegyuides, Applied Physics Letters 95, 2007082507 